\title{
Phosphorus in kidney disease: Culprit or bystander?
}

$\mathbf{P}$ HOSPHORUS IS ESSENTIAL for life. However, both low and high levels of phosphorus in the body have consequences, and its concentration in the blood is tightly regulated through dietary absorption, bone flux, and renal excretion and is influenced by calcitriol $\left(1,25\right.$ hydroxyvitamin $\left.\mathrm{D}_{3}\right)$, parathyroid hormone, and fibroblast growth factor 23 (FGF23).

See related articles, page 584 and page 629

Sekar et al, ${ }^{1}$ in this issue of the Journal, provide an extensive review of the pathophysiology of phosphorus metabolism and strategies to control phosphorus levels in patients with hyperphosphatemia and end-stage kidney disease.

\section{PHOSPHORUS OR PHOSPHATE?}

What's in a name? That which we call a rose By any other word would smell as sweet.

-Shakespeare, Romeo and Juliet

The terms phosphate and phosphorus are often used interchangeably, though most writers still prefer phosphate over phosphorus.

The serum concentrations of phosphate and phosphorus are the same when expressed in millimoles per liter, as every mole of phosphate contains 1 mole of phosphorus, but not the same when expressed in milligrams per deciliter. ${ }^{2}$ The molecular weight of phosphorus is 30.97, whereas the molecular weight of the phosphate ion $\left(\mathrm{PO}_{4}{ }^{3-}\right)$ is 94.97 -more than 3 times higher. Therefore, using these terms interchangeably in this context can lead to numerical error. ${ }^{3}$

doi:10.3949/ccjm.85a.18013
Phosphorus, being highly reactive, does not exist by itself in nature and is typically present as phosphates in biologic systems. When describing phosphorus metabolism, the term phosphates should ideally be used because phosphates are the actual participants in the bodily processes. But in the clinical laboratory, all methods that measure serum phosphorus in fact measure inorganic phosphate and are expressed in terms of milligrams of phosphorus per deciliter rather than milligrams of phosphate per deciliter, and using these 2 terms interchangeably in clinical practice should not be of concern. ${ }^{4}$

\section{THE PROBLEM}

US adults typically ingest $1,200 \mathrm{mg}$ of phosphorus each day, and about $60 \%$ to $70 \%$ of the ingested phosphorus is absorbed both by passive paracellular diffusion via tight junctions and by active transcellular transport via sodium-phosphate cotransport. The kidneys must excrete the same amount daily to maintain a steady state. As kidney function declines, phosphorus accumulates in the blood, leading to hyperphosphatemia.

Hyperphosphatemia is often asymptomatic, but it can cause generalized itching, red eyes, and adverse effects on the bone and parathyroid glands. Higher serum phosphorus levels have been shown to be associated with vascular calcification, ${ }^{5}$ cardiovascular events, and higher all-cause mortality rates in the general population, ${ }^{6}$ in patients with diabetes, ${ }^{7}$ and in those with chronic kidney disease. ${ }^{8}$ This association between higher serum phosphorus levels and the all-cause mortality rate led to the assumption that lowering serum phospho-
As kidney function declines, phosphorus accumulates in the blood 
TABLE 1

Phosphorus binders

Calcium-based
Calcium carbonate
Calcium acetate
Noncalcium-based
Metal-based
Aluminum hydroxide (only for short-term use)
Lanthanum carbonate
Iron (sucroferric oxyhydroxide, ferric citrate)
Resin-based
Sevelamer (sevelamer hydrochloride,
sevelamer carbonate)
Others
Magnesium hydroxide, magnesium carbonate
(risk of hypermagnesemia)
Chitosan (minimal effect)

rus levels in these patients could reduce the rates of cardiovascular events and death, and to efforts to correct hyperphosphatemia.

Research into FGF23 continues, especially its role in cardiovascular complications of chronic kidney disease, as both phosphorus and FGF23 levels are elevated in chronic kidney disease and are implicated in poor clinical outcomes in these patients. However, both FGF23 and parathyroid hormone levels rise early in the course of kidney disease, long before overt hyperphosphatemia develops. Further, FGF23 rises earlier than parathyroid hormone and has been found to be an independent risk factor for cardiovascular events and death from any cause in end-stage kidney disease. ${ }^{9}$

Whether hyperphosphatemia is the culprit or merely an epiphenomenon of metabolic complications of chronic kidney disease is still unclear, as more molecules are being identified in the complex process of cardiovascular calcification. ${ }^{10}$

However, one thing is clear: vascular calcification is not just a simple precipitation of calcium and phosphorus. Instead, it is an active process that involves many regulators of mineral metabolism. ${ }^{10}$ The complex nature of this process is likely one of the reasons that evidence is conflicting ${ }^{11}$ about the benefits of phosphorus binders in terms of cardiovascular events or all-cause mortality in these patients.

\section{STRATEGIES TO CONTROL HYPERPHOSPHATEMIA}

\section{Reducing intake}

Dietary phosphorus restriction is the first step in controlling serum phosphorus. But reducing phosphorus intake while otherwise trying to optimize the nutritional status can be challenging.

The recommended daily protein intake is 1.0 to $1.2 \mathrm{~g} / \mathrm{kg}$. But phosphorus is typically found in foods rich in proteins, and restricting protein severely can compromise nutritional status and may be as bad as elevated phosphate levels in terms of outcomes.

Although plant-based foods contain more phosphate per gram of protein (ie, they have a higher ratio of phosphorus to protein) than animal-based foods, the bioavailability of phosphorus from plant foods is lower. Phosphorus in plant-based foods is mainly in the form of phytate. Humans cannot hydrolyze phytate because we lack the phytase enzyme; hence, the phosphorus in plant-based foods is not well absorbed. Therefore, a vegetarian diet may be preferable and beneficial in patients with chronic kidney disease. A small study in humans showed that a vegetarian diet resulted in lower serum phosphorus and FGF23 levels, but the study was limited by its small sample size. ${ }^{12}$

Patients should be advised to avoid foods that have a high phosphate content, such as processed foods, fast foods, and cola beverages, which often have phosphate-based food additives.

Further, one should be cautious about using supplements with healthy-sounding names. A case in point is "vitamin water": $12 \mathrm{oz}$ of this fruit punch-flavored beverage contains $392 \mathrm{mg}$ of phosphorus, ${ }^{13}$ and this alone would require 12 to 15 phosphate binder tablets to bind its phosphorus content.

In addition, many prescription drugs have significant amounts of phosphorus, and this is often unrecognized.

Sherman et $\mathrm{al}^{14}$ reviewed 200 of the most commonly prescribed drugs in dialysis patients and found that $23(11.5 \%)$ of the drug labels listed phosphorus-containing ingredients, but the actual amount of phosphorus was not listed. The phosphorus content ranged from 1.4 mg (clonidine $0.2 \mathrm{mg}$, Blue Point Laboratories, Dublin, Ireland) to $111.5 \mathrm{mg}$ (paroxetine 40 mg, GlaxoSmith Kline, Philadelphia, PA). 
The phosphorus content was inconsistent and varied with the dose of the agent, type of formulation (tablet or syrup), branded or generic formulation, and manufacturer.

Branded lisinopril (Merck, Kenilworth, NJ) had $21.4 \mathrm{mg}$ of phosphorus per 10-mg dose, while a generic product (Blue Point Laboratories, Dublin, Ireland) had $32.6 \mathrm{mg}$. Different brands of generic amlodipine $10 \mathrm{mg}$ varied in their phosphorus content from $8.6 \mathrm{mg}$ (Lupin Pharmaceuticals, Mumbai, India) to $27.8 \mathrm{mg}$ (Greenstone LLC, Peapack, NJ) to $40.1 \mathrm{mg}$ (Qualitest Pharmaceuticals, Huntsville, AL. Rena-Vite (Cypress Pharmaceuticals, Madison, MS), a multivitamin marketed to patients with kidney disease, had $37.7 \mathrm{mg}$ of phosphorus per tablet. Thus, just to bind the phosphorus content of these 3 tablets (lisinopril, amlodipine, and Rena-Vite), a patient could need at least 3 to 4 extra doses of phosphate binder.

The phosphate content of medications should be considered when prescribing. For example, Reno Caps (Nnodum Pharmaceuticals, Cincinnati, $\mathrm{OH}$ ), another vitamin supplement, has only $1.7 \mathrm{mg}$ of phosphorus per tablet and should be considered, especially in patients with poorly controlled serum phosphorus levels. However, the challenge is that medication labels do not provide the phosphorus content.

\section{Reducing phosphorus absorption}

Because so many foods contain phosphorus, dietary efforts alone are often insufficient to control serum phosphorus levels, and most patients require additional strategies, eg, phosphorus binders (Table 1).

Although these agents reduce serum phosphorus and help reduce symptoms, an important quality-of-life measure, it is uncertain whether they improve clinical outcomes. ${ }^{11}$ To date, no specific phosphorus binder offers a survival benefit over placebo. ${ }^{11}$

Based on the limited and conflicting evidence, the Kidney Disease: Improving Global Outcomes (KDIGO) guidelines, recently updated, suggest that oral phosphorus binders should be used in patients with hyperphosphatemia to lower serum phosphorus levels toward the normal range..$^{15}$ They further recommend not exceeding $1,500 \mathrm{mg}$ of elemental calcium per day if a calcium-based binder is used,

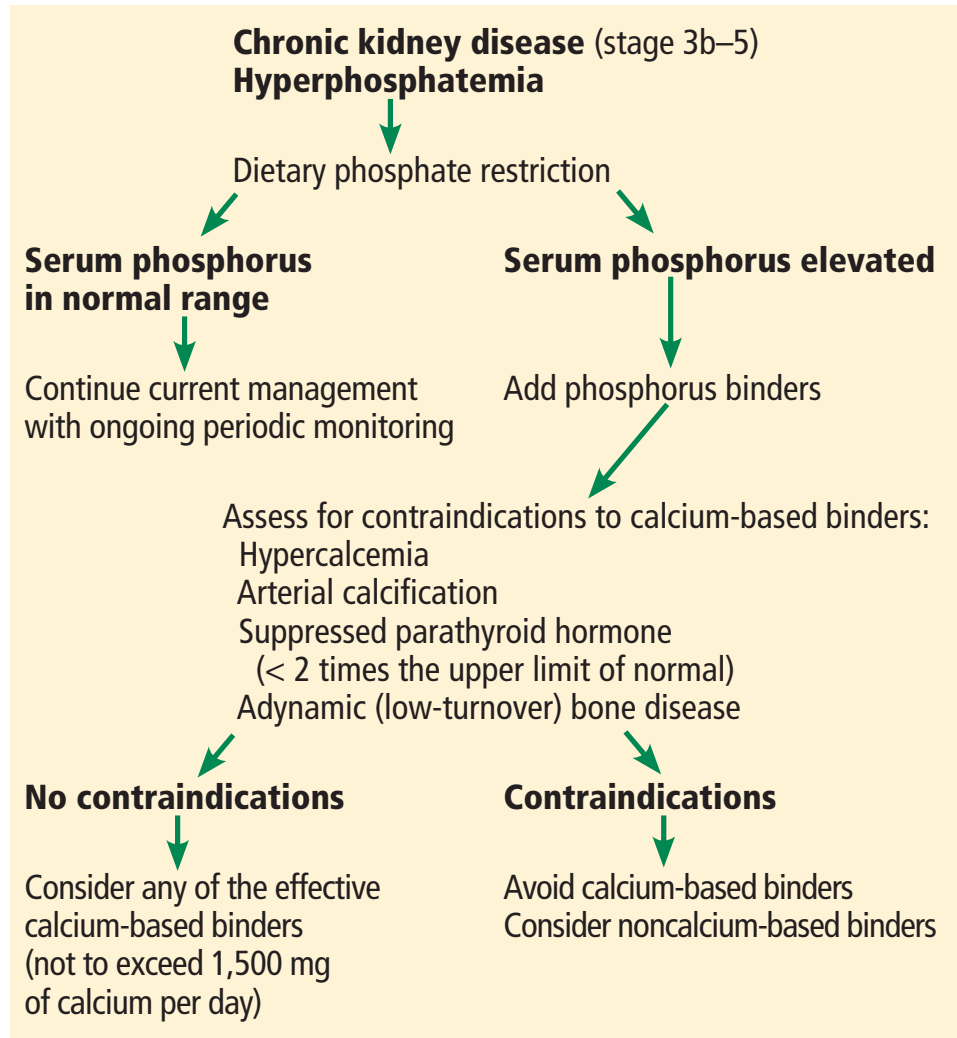

Figure 1. A stepwise approach to the management of hyperphosphatemia and selection of phosphorus binder.

and they recommend avoiding calcium-based binders in patients with hypercalcemia, adynamic bone disease, or vascular calcification.

Phosphorus binders may account for up to $50 \%$ of the daily pill burden and may contribute to poor medication adherence. ${ }^{16}$ Dialysis patients need to take a lot of these drugs: by weight, 5 to 6 pounds per year.

These drugs can bind and interfere with the absorption of other vital medications and so should be taken with meals and separately from other medications.

At present, there is insufficient evidence to recommend one binder over the other, and the selection of phosphorus binder should be individualized for each patient, taking into consideration the stage of chronic kidney disease, degree of hyperphosphatemia, concomitant anemia, presence of vascular calcification, use of other medications, side effects, cost to the individual, and pill burden. A stepwise, opinion-based, clinical approach to the selection of the phosphorus binders in patients with hyperphosphatemia is presented in Figure 1. 


\section{Removing phosphorus}

Removal of phosphorus by adequate dialysis or kidney transplant is the final strategy.

\section{New agents under study}

To improve phosphorus control, other agents that inhibit absorption of phosphate are being investigated.

Nicotinamide reduces expression of the sodium-phosphorus cotransporter NTP2b. Its use in combination with a low-phosphorus diet and phosphorus binders may maximize reductions in phosphorus absorption and is being studied in the CKD Optimal Management With Binders and Nicotinamide (COMBINE) study.

Tenapanor, an inhibitor of the sodiumhydrogen transporter NHE3, has been shown in animal studies to increase fecal phosphate excretion and decrease urinary phosphate excretion ${ }^{17}$ but requires further evaluation.

\section{REFERENCES}

1. Sekar A, Kaur T, Nally JV Jr, Rincon-Choles H, Jolly S, Nakhoul G. Phosphorus binders: the new and the old, and how to choose. Cleve Clin J Med 2018; 85(8):629-638. doi:10.3949/ccjm.85a.17054

2. Young DS. "Phosphorus" or "phosphate." Ann Intern Med 1980; 93(4):631. pmid:7436198

3. Bartter FC. Reporting of phosphate and phosphorus plasma values. Am J Med 1981; 71(5):848. PMID:7304659.

4. Iheagwara OS, Ing TS, Kjellstrand CM, Lew SQ. Phosphorus, phosphorous, and phosphate. Hemodial Int 2013; 17(4):479-482. doi:10.1111/hdi.12010

5. Adeney KL, Siscovick DS, Ix JH, et al. Association of serum phosphate with vascular and valvular calcification in moderate CKD. J Am Soc Nephrol 2009; 20(2):381-387. doi:10.1681/ASN.2008040349

6. Dhingra R, Sullivan LM, Fox CS, et al. Relations of serum phosphorus and calcium levels to the incidence of cardiovascular disease in the community. Arch Intern Med 2007; 167(9):879-885. doi:10.1001/archinte.167.9.879

7. Chonchol M, Dale R, Schrier RW, Estacio R. Serum phosphorus and cardiovascular mortality in type 2 diabetes. Am J Med 2009; 122(4):380 386. doi:10.1016/j.amjmed.2008.09.039

8. Covic A, Kothawala P, Bernal M, Robbins S, Chalian A, Goldsmith D. Systematic review of the evidence underlying the association between mineral metabolism disturbances and risk of all-cause mortality, cardiovascular mortality and cardiovascular events in chronic kidney disease. Nephrol Dial Transplant 2009; 24(5):1506-1523. doi:10.1093/ndt/gfn613

9. Gutiérrez OM, Mannstadt M, Isakova T, et al. Fibroblast growth factor 23 and mortality among patients undergoing hemodialysis. N Engl J Med 2008; 359(6):584-592. doi:10.1056/NEJMoa0706130

10. Lullo LD, Barbera V, Bellasi A, et al. Vascular and valvular calcifications in chronic kidney disease: an update. EMJ Nephrol 2016; 4(1):84-91. https://pdfs.semanticscholar.org/150f/c7b5dfe671c9b61e4c76d54b- 7d713b60ba6a.pdf. Accesssed June 5, 2018.

11. Palmer SC, Gardner S, Tonelli M, et al. Phosphate-binding agents in adults with CKD: a network meta-analysis of randomized trials. Am J Kidney Dis 2016; 68(5):691-702. doi:10.1053/j.ajkd.2016.05.015

12. Moe SM, Zidehsarai MP, Chambers MA, et al. Vegetarian compared with meat dietary protein source and phosphorus homeostasis in chronic kidney disease. Clin J Am Soc Nephrol 2011; 6(2):257-264. doi:10.2215/CJN.05040610

13. Moser M, White K, Henry B, et al. Phosphorus content of popular beverages. Am J Kidney Dis 2015; 65(6):969-971. doi:10.1053/j.ajkd.2015.02.330

14. Sherman RA, Ravella S, Kapoian T. A dearth of data: the problem of phosphorus in prescription medications. Kidney Int 2015; 87(6):10971099. doi:10.1038/ki.2015.67

15. KDIGO 2017 clinical practice guideline update for diagnosis, evaluation, and treatment of chronic kidney disease-mineral and bone disorder (CKD-MBD). Kidney Int Supplements 2017; 7(1 suppl): 1-59. www.kisupplements.org/article/S2157-1716(17)30001-1/pdf. Accessed June 5, 2018.

16. Fissell RB, Karaboyas A, Bieber BA, et al. Phosphate binder pill burden, patient-reported non-adherence, and mineral bone disorder markers: findings from the DOPPS. Hemodial Int 2016; 20(1):38-49. doi:10.1111/hdi.12315

17. Labonté ED, Carreras CW, Leadbetter MR, et al. Gastrointestinal inhibition of sodium-hydrogen exchanger 3 reduces phosphorus absorption and protects against vascular calcification in CKD. J Am Soc Nephrol 2015; 26(5):1138-1149. doi:10.1681/ASN.2014030317

ADDRESS: Malvinder S. Parmar, MB, MS, Internal Medicine, Timmins and District Hospital, 700 Ross Ave. East, Timmins, Ontario P4N 8P2 Canada; Wittykidney@outlook.com 